\title{
The unsolved mystery of apoA-I recycling in adipocyte
}

\author{
Shuai Wang ${ }^{1}$, Dao-quan Peng ${ }^{1^{*}}$ and Yuhong Yi ${ }^{2}$
}

\begin{abstract}
As the major storage site for triglycerides and free cholesterol, adipose tissue plays a central role in energy metabolism. ApoA-I is the main constituent of HDL and plays an important role in removal of excess cholesterol from peripheral tissues. Recently, multiple studies have shown beneficial effects of apoA-I on adipose metabolism and function. ApoA-I was reported to improve insulin sensitivity and exert anti-inflammatory, anti-obesity effect in animal studies. Interestingly, Uptake and resecretion of apoA-I by adipocytes has been detected. However, the significance of apoA-I recycling by adipocytes is still not clear. This article reviewed methods used to study cellular recycling of apoA-I and summarized the current knowledge on the mechanisms involved in apoA-I uptake by adipocytes. Since the main function of apoA-I is to mediate reverse cholesterol transport from peripheral tissues, the role of apoA-l internalization and re-secretion by adipocytes in intracellular cholesterol transport under physiological and pathological conditions were discussed. In addition, findings on the correlation between apoA-I recycling and obesity were discussed. Finally, it was proposed that during intracellular transport, apoA-I-protein complex may acquire cargoes other than lipids and deliver regulatory information when they were resecreted into the plasma. Although apoA-I recycling by adipocytes is still an unsolved mystery, it's likely that it is more than a redundant pathway especially under pathological conditions.
\end{abstract}

Keywords: Lipoprotein, Adipocytes, Uptake, Resecretion, Cholesterol

\section{Background}

Apolipoprotein A-I (apoA-I) is synthesized and secreted by the liver and intestine. As the major protein constituent of high-density lipoprotein (HDL), apoA-I plays an important role in reverse cholesterol transport from peripheral tissues to the liver [1]. As the body's largest reservoir of free cholesterol, adipose tissue contributes to apoA-I lipidation and nascent HDL biogenesis [2, 3]. On the other side, apoA-I has been reported to have reciprocal effects on adipose tissue metabolism and function. For example, apoA-I has been reported to promote glucose uptake [4], improve insulin sensitivity [5], upregulate the expression of adiponectin [6], and exert anti-inflammatory effect [7, 8]. It is generally accepted that the multiple effects of apoA-I is mediated through regulation of lipid raft or direct binding with surface receptors, resulting in subsequent activation of intracellular signaling $[9,10]$. What is interesting is our previous

\footnotetext{
* Correspondence: pengdq@hotmail.com

'Department of Cardiology, The Second Xiangya Hospital of Central South

University, Changsha, Hunan, China

Full list of author information is available at the end of the article
}

finding that apoA-I protein was found in human adipose tissue while apoA-I mRNA was not detected, indicating that apoA-I found in adipose tissue was exogenous. Actually, cellular internalization of apoA-I has been observed in several cell lines, including macrophages, endothelium and smooth muscle cells [11-13]. However, it is not until recent years that the uptake and resecretion of apoA-I by adipocytes has been confirmed with the significance still remaining unclear $[14,15]$. This article briefly discussed methods used to study apoA-I recycling, mechanisms for apoA-I internalization and the possible significance of apoA-I recycling by adipocytes.

\section{ApoA-I-induced cell signaling and adipocyte metabolism} Except for mediating reverse cholesterol transport, there are several well documented evidence suggesting that apoA-I could regulate cellular function of adipocytes. ApoA-I has been reported to exert anti-inflammatory effect on adipocytes. ApoA-I reduced monocyte chemotactic protein-1 (MCP-1) and serum amyloid A3 (SSA3) expression and attenuated reactive oxygen species (ROS) 
through diminishing the translocation of NADPH oxidase (nicotinamide adenine dinucleotide phosphateoxidase) into lipid rafts [7]. In vitro study showed that in cultured 3T3-L1 adipocytes apoA-I upregulated the expression of adiponectin in phosphatidylinositol 3-kinase (PI3K)-dependent manner [6]. In brown adipocytes, apoA-I upregulated the expression of uncoupling protein 1 , which participated in the control of energy expenditure, possibly involved Adenosine 5'-monophosphateactivated protein kinase (AMPK) signaling activation [16]. It is so far generally accepted that the multiple effect of apoA-I are results of intracellular signaling cascade triggered either through a direct interaction between apoA-I and surface receptors such as ATP-binding cassette transporter A1 (ABCA1) and $\beta$ subunit of ATP synthase ( $\beta$ ATPase) [17-19] or through lipid raft disruption resulted from apoAI-ABCA1 complex induced cholesterol efflux [9, 10]. Although the phenomenon of apoA-I internalization has been observed in vascular cells such as macrophage and endothelium, the physiological significance remains undetermined. Besides, uptake and resecretion of apoA-I by adipocytes has only been demonstrated in recent years.

\section{Methods to study the recycling of ApoA-I by adipocytes}

Studies in intracellular transport of lipoproteins demand specific and robust labeling of the target entity with minimal alteration of the investigated transport process by the labeling strategy. To achieve this goal, recombinant apoA-I needs to be constructed so that the modified apoA-I can bind with the labeling probe without affecting its main biological properties. Pulse-chase experiments of isotope- or fluorescence-labeled proteins are the usual approach used to gather dynamic information about apoA-I internalization and resecretion. Confocal studies of labeled apoA-I and labeled markers of specific organelles or proteins can give information about apoAI localization and possible interaction with other proteins.

Fluorescent labeling was used to address the cellular uptake of apoA-I, which relies on labeling of a recombinant form of apoA-I with a fluorescent probe at a single and highly targeted site in the molecule without altering the main function of apoA-I (the ability to promote cholesterol efflux). Because human apoA-I ordinarily lacks a cysteine, which is necessary for a thiol-reactive probe (e.g. agent Alexa Fluor 546 C5maleimide) to de attached, recombinant forms of apoAI needs to be constructed. Cys residue needs to be introduced without affecting the ability of apoA-I to promote cholesterol efflux.

In humans, apoA-I is synthesized in the liver and intestinal cells as a non-glycosylated pre-pro-protein [20]. The 18 amino acid pre-segment is removed before the protein leaves the cell whilst the 6 amino acid pro-segment is cleaved during post secretion, leaving the mature 243 amino acid protein [21]. Since apoA-I is non-glycosylated, bacterial expression system such as Escherichia coli is usually the preferred option because eukaryotic systems (e.g. Chinese hamster ovary cell system, $\mathrm{CHO}$ ) are generally more difficult to transfect than bacteria. Besides, once successfully transfected, $\mathrm{CHO}$ cell colonies must undergo an in-depth screening process to find stably transfected, high expression colonies. In addition, eukaryotic cells require a longer period of time for expression and a much higher level of maintenance than bacteria. However, if the prosegment of apoA-I was removed, expression level in Escherichia coli would be very low unless one modifies the existing DNA sequence for the first 8 amino acid to a sequence in which bacterial codons are more readily available [22]. Therefore, fusion proteins containing a histidine tag which can be purified over a nickel chelating column have been created for the purpose of both expression and purification. Before using these expression products in experiments, the His tag sequence needs to be proteolytically cleaved away from the target sequence because it may adversely affect both structure and function of the apoA-I. Enteropeptides [23], factor Xa [24], and thrombin [25] are often used proteolytic systems and work well for many proteins. However, their use for apolipoprotein systems are limited by the fact that these proteases often cut at locations within the target protein in addition to the intended site engineered between the fusion and target proteins. In the case of apoA-I, this problem has been circumvented by lipidating the protein before cleavage, masking the secondary cleavage sites through a conformational change [26]. Another method to address this problem was IgA protease system. It was found that IgA protease from Neisseria gonorrhoeae did not cleave the wild type apoA-I sequence [27]. Therefore, adding an Igase site to the pET30 expression vector containing the apoA-I cDNA, IgA protease cleaved the His-tag without affecting the target apoA-I product [27]. As an alternative to proteasedependent removal of the N-terminal His-Tag, an acid labile Asp-Pro peptide bond was introduced into the apoA-I sequence between amino acids 2 and 3 of the wild type apoA-I sequence, which was achieved by mutating Glu2 to Asp, taking advantage of the presence of the Pro at position 3 in wild type apoA-I. Formic acid could efficiently cleave the apoA-I His-Tag at this acid labile peptide bond without exerting discernable effect on the apoA-I product, presenting as an efficient way for protein purification [28]. Fluorescent microscopy studies 
were then used to follow the cell biology of fluorescent labeled apoA-I.

Although fluorescent microscopy is well suited for measuring fluorescent apoA-I internalization, it gives little information on the fate of the label after internalization. Therefore, pulse-chase experiment through radiolabeled apoA-I could be used to study the fate of the internalized protein. Because nonspecific radioiodination of apoA-I can have significant effect on its structure and metabolism [29], endogenous labeling of wild-type apoA-I expressed in methionine auxotrophline line of bacteria (E. coli) by ${ }^{35} \mathrm{~S}$-methionine is nowadays a commonly accepted method [12].

Although approaches based on the use of fluorescent labeled or radiolabeled apoA-I are highly valuable to study lipoprotein uptake, it is necessary to realize that there are some limitations due to unspecific labeling of cellular compartment caused by partial degradation of apoA-I followed by release of the small labeling probe. Besides, there is difficulty in distinguishing cellular incorporation of the protein from protein absorption to plasma membrane.

To solve the problem, a method was developed which could provide an unambiguous proof of apoA-I recycling by adipocytes through creating a functional recombinant apoA-I containing a phosphorylation site (pka-apoA-I), which could be recognized by the catalytic subunit of cAMP-dependent protein kinase (PKA) [15]. Because cellular uptake of the protein would allow its phosphorylation and the phosphorylated protein would be found in the cell culture medium if re-secreted, phosphorylated apoA-I in the medium was correlated to the extent of apoA-I recycling. pka-apoA-I construct was prepared by sub-cloning the full-length sequence of mature human apoA-I into a commercial vector that incorporated an $\mathrm{N}$-terminal tag encoding for a six-His tag and a five amino acid recognition sequence (RRASV) for the catalytic subunit of PKA. Adipocytes were pre-incubated with ${ }^{32} \mathrm{P}$-phosphate to radiolabel the cellular pool of ATP. Phosphorylated apoA-I collected from the culture medium after different times of incubation with pkaapoA-I were subjected to SDS-PAGE, followed by coomassie blue staining and autoradiography to analyze the recycling of apoA-I by adipocytes $[14,15]$.

\section{Mechanism of ApoA-I uptake by adipocytes Receptor mediated process}

ApoA-I entering into adipocytes may be a receptor mediated process or the result of nonspecific endocytic membrane invagination. In line with several other cell types [12], a receptor mediated process was supported by the results that the content of phosphorylated pkaapoA-I in the culture medium, which represented the rate of apoA-I recycling, increased in response to increase in concentration of recombinant apoA-I (pkaapoA-I) and approached a plateau at concentrations higher than $75 \mu \mathrm{g} / \mathrm{ml}$. Besides, human apoA-I purified from plasma reduced apoA-I recycling in a dose dependent manner, indicating a competition between human and recombinant apoA-I for binding to a common receptor [14].

\section{Clathrin}

While LDL endocytosis occurs via clathrin-coated pits [30], it is unclear whether apoA-I internalization is clathrin dependent. It was shown that blocking clathrinmediated endocytosis by its specific inhibitor monodansyl cadaverine (MDC) abolished apoA-I internalization into macrophage (RAW and THP-1 cell), suggesting uptake of apoA-I by macrophages is clathrin-dependent $[13,31]$. However, considering heterogeneity may exist between different tissues and cell lines, further study need to be carried out to clarify if clathrin-pathway is involved in apoA-I uptake by adipocytes.

\section{Caveolae}

Caveolae, implicated in a variety of physiological process including cell signaling and endocytosis, are curved lipid raft regions rich in cholesterol and sphingolipids [32]. Caveolin-1, which is the main protein constituent of caveolae, has been shown to interact with $A B C A 1$ and is involved in cholesterol efflux to apoA-I in several cell types [33]. Direct interaction of caveolin-1 with apoA-I has also been reported. Mouse embryonic fibroblasts (MEFs) derived from wild type animals were reported to have 2.6-fold more apoA-I binding sites than MEFs derived from caveolin-1 deficient animals. In addition, caveolin-1 binding with apoA-I was reported to target apoA-I for internalization [34]. Considering the remarkably abundant caveolae on the surface of adipocytes which accounts for $50 \%$ of the surface area [32], it is speculated that caveolae may be involved in apoA-I uptake by adipocytes. Whether caveolae is pre-requisite for apoA-I internalization and whether the presence of caveolae accounts for a favorable environment for apoA-I internalization warrant further study.

\section{ABCA1 independent process}

Because apoA-I could directly bind with ABCA1 which rapidly shuttles between intracellular compartment and plasma membrane [35], whether apoA-I internalization is ABCA1 dependent has been explored. Previous studies which focused on macrophages observed co-localization of apoA-I and ABCA1 in endosomal compartment [36-38], supporting the idea that ABCA1-apoA-I complex were delivered to intracellular compartment where it received lipids and formed a nascent-HDL like particles that were subsequently secreted from 
the cell. However, a recent study carried out in adipocytes showed that Brefeldin A (BFA), which inhibited vesicular trafficking of ABCA1, didn't affect apoA-I recycling by adipocytes, suggesting an $\mathrm{ABCA} 1$ independent way for apoA-I uptake may exist [14].

\section{Mediated by $\beta$-ATPase}

$\beta$-ATPase mainly presents in mitochondria membrane and is the terminal enzyme of the oxidative phosphorylation pathway. Unexpectedly, ectopic localization of $\beta$ ATPase on cell surface were found in several cells types including hepatocytes and adipocytes. What's intriguing is that $\beta$-ATPase has been characterized as a receptor for apoA-I $[15,17,39]$. The binding of apoA-I to $\beta$ ATPase induced ATP hydrolysis and promoted extracellular ADP generation, which in turn stimulated intracellular signaling by activating purinergic receptors, which belong to the family of G-Protein-coupled receptors stimulated by extracellular nucleotides. In hepatocytes, it was demonstrated the interaction of the $\beta$-ATPase with apoA-I modulated HDL endocytosis [17, 40]. Niacin, which is used to raise plasma levels of HDL, has been shown to reduce the ectopic expression of the $\beta$-ATPase in hepatocytes and inhibit cellular uptake of HDL [41]. In adipocytes, $\beta$-ATPase has been proven to be involved in apoA-I uptake, evidenced by the fact that apoA-I recycling was blocked by a monoclonal antibody against the $\beta$-ATPase [15].

\section{Regulation of apoA-I internalization through CAMP-PKA signaling}

Previous studies revealed that the interaction between apoA-I and ABCA1 activated adenylate cyclase and increased the content of cAMP [42]. cAMP/protein kinase A (PKA) were reported to play an important role in regulating apoA-I/ABCA1-induced lipid translocation and removal. Pharmacologic modulation to decrease cAMP levels and PKA activity led to reduction in apoAI-ABCA1 mediated cholesterol efflux [43, 44]. Conversely, interference to activate cAMP/PKA signaling elevated apoA-I-induced cholesterol efflux [44]. The molecular mechanisms may include: 1) regulation of ABCA1 expression by cAMP/PKA signaling at the transcriptional level [42], 2) phosphorylation of ABCA1 by PKA may result in altering the conformation of the protein to a more active state for lipid translocation across the cell membrane [45]. However, an interesting phenomenon was observed in the murine macrophages RAW 264 cell that cholesterol efflux to apoA-I was absent in the absence of cAMP but inducible by cAMP analogues 8-Br-cAMP [31, 46]. Coincidently, compared with cAMP treatment, cellular uptake and resecretion of apoA-I was apparently much less in the basal condition when cAMP is absent [31, 46]. Based on these interesting findings, it was queried was cellular internalization of apoA-I pre-requisite for apoA-I-induced cholesterol efflux or were they just two independent events subsequent to apoA-I binding with surface receptor? Based on previous studies carried out in macrophages, it is generally accepted that the contribution of internalized apoA-I to cholesterol efflux and HDL formation seems to be limited [12, 47].

In adipocytes, cAMP/PKA signaling was demonstrated to regulate apoA-I recycling. Stimulation with isoproterenol, which raised cAMP and activated PKA, accelerated recycling of apoA-I by 3 T3-L1 adipocytes. PKA inhibitor H89 inhibited the internalization and resecretion of apoA-I $[14,15]$. The molecular mechanism underlying the regulatory effect of cAMP-PKA signaling on the recycling of apoA-I by adipocytes remains unclear. Whether PKA would regulate the binding ability of apoA-I with surface $\beta$-ATPase, which is involved in mediating apoA-I internalization, is not known. In addition, whether PKA may regulate the expression of $\beta$-ATPase or its translocation to the plasma membrane need to be further investigated.

\section{Physiological role of ApoA-I internalization in adipocytes ApoA-I internalization and adipocyte cholesterol efflux}

The physiological significance of apoA-I internalization in adipocytes is not known. As mentioned above, the mechanism of lipid-free apoA-I lipidation is not fully understood yet and whether apoA-I lipidation may require its internalization remains controversial. Some studies supported the idea that apoA-I lipidation mainly occurred at the cell surface. A model by Phillips proposed that ABCA1 shuttled phospholipids from the inner to extracellular surface of the plasma membrane, resulting in membrane bulges with high curvature that were sufficient to allow apoA-I penetration. Once apoAI bound to ABCA1 on the cell surface and was lipidated, the N-terminus of apoA-I was unfolded and formed an unstable intermediate structure that was rapidly released from the cell $[48,49]$. On the other hand, other studies reported that apoA-I internalization was involved in apoA-I induced cholesterol efflux. In previous reported studies, RAW cells were cholesterol loaded, incubated with apoA-I and treated with 8-Br-cAMP to stimulate the take up of apoA-I. After indicated period of treatment, apoA-I was depleted from the culture medium through washing. Significant cAMP-dependent $\left[{ }^{3} \mathrm{H}\right]$ cholesterol efflux was detected during chase period even in the absence of exogenous apoA-I and HDL in the culture medium. Besides, the degree of cholesterol efflux was positively correlated to the extent of apoA-I internalization, indicating cholesterol efflux detected in this study was due to re-secretion of the internalized and lipidated apoA-I [31]. 
As the body's largest pool of free cholesterol, adipose tissue was demonstrated to release cholesterol and contribute to HDL biogenesis [2, 3, 50]. Is apoA-I internalization involved in adipocytes cholesterol release? First of all, internalization of apoA-I is not indispensable for adipocytes cholesterol release because blocking apoA-I internalization does't have a significant effect on cholesterol efflux [15]. However, it is worth noting that under basal condition cellular uptake of apoA-I is limited. Therefore, it may be more appropriate to consider the significance of apoA-I internalization under circumstances when the degree of apoA-I internalization is more prominent.

\section{Intracellular cholesterol transport during cholesterol mobilization}

It is interesting to note that both apoA-I internalization and adipocyte lipolysis are stimulated by PKA activation. Multiple evidence suggested that cholesterol and triglyceride homeostasis were coupled in adipocytes [51, 52], which led to the question what the role of apoA-I in intracellular cholesterol transport during lipid mobilization may be. Adipose is the body's largest pool for free cholesterol. In adipocytes, $<6 \%$ cholesterol stored is in the esterified from [53,54], which is different from other cell type [55]. Surface layer of lipid droplet was identified as primary intracellular storage site for free cholesterol within adipocytes [56]. It was shown in 3 T3-L1 adipocytes that under basal conditions cholesterol mobilization from adipocytes onto apoA-I was very limited despite induction of ABCA1 expression. However, under conditions of sustained lipolytic stimulation, a boost in the mobilization of adipocyte cholesterol was observed [52]. Besides, stimulation of lipolysis is accompanied by an increased cholesterol transport from surface of lipid droplet to plasma membrane (PM) where cholesterol can be removed [51]. Generally, there are vesicular and nonvesicular ways through which the highly hydrophobic free cholesterol can be transported from the lipid droplet pool to PM-pool. Cholesterol could be present in the membrane of intracellular vesicles for trafficking, which requires intact cytoskeleton and ATP to provide moving tracks and energy. Nonvesicular transport is mediated by diffusible carrier proteins, which have hydrophobic cavities to bind cholesterol and transport it across the aqueous cytosol $[57,58]$. Cholesterol, together with phospholipids and proteins, were reported to be incorporated into cytosolic lipid protein particles (CLPP) which were HDL-like cytosolic lipidprotein complex with density of $1.09-1.16 \mathrm{~g} / \mathrm{ml}$ and diameters of 17-18 nm [59]. In order to transport cholesterol to the target cellular compartment, the cholesterol transfer protein in CLPP needs to contain a specific membrane contact site. For example, intracellular transport from late endosome to mitochondria involves steroidogenic acute regulatory (StAR) protein on the mitochondria and cholesterol donating StAR-related lipid transfer (START) domain protein 3 (StARD3) on late endosomes $[60,61]$. In the case of cholesterol transport from the ER (endoplasmic reticulum) to Golgi, cholesterol transport depends on the activity of oxysterol binding protein (OSBP) to create membrane contact sites between both organelles [62,63]. What's interesting is that caveolin-1, a critical protein found in membrane domain caveolae, has been found as protein constituent of CLPP. Since direct interaction of apoA-I with caveolin-1 has been confirmed at the cell surface [34], it would be interesting to study intracellular interaction of these two proteins and its possible role in intracellular cholesterol transport. It is speculated that intracellular apoA-I may be involved in CLPP formation, facilitating intracellular cholesterol transport to plasma membrane for further removal through efflux pathway. Therefore, apoA-I internalization and resecrestion by adipocytes may be accompanying process of cholesterol mobilization and represent an alternative pathway to maintain cholesterol homeostasis.

\section{Relevance to obesity}

Recently, the possible anti-obesity effect of apoA-I has triggered great interest. In mouse models, apoA-I transgenic mice had significant lower fat content than wild type mice after feeding with high fat diet for three months [16]. In other studies, daily administration of apoA-I mimetics D-4 F and L-4 F in high fat diet fed mice reduced weight gain and decreased obesity when compared with age-matched vehicle-treated obese mice $[5,64]$. Several studies demonstrated that apoA-I may contribute to modulating body fat content by controlling the extent of lipolysis $[5,65]$. Recent study showed that the naturally occurring apoA-I variant Milano, which contains a single point mutation that lead to an Arg (173) Cys substitution, reduced fat mass through stimulation of lipolysis. Similar to apoA-I WT, the anti-obesity effect of Milano was independent of ABCA1 nor the canonical CAMP/PKA signaling pathway [66]. Interestingly, the Milano-stimulated lipolysis was much greater compared with apoA-I WT [66]. Since apoA-I Milano was demonstrated to be more efficient in modulating cholesterol efflux than apoA-I WT [67], this finding further supported the notion that cholesterol mobilization was coupled with lipolysis. It would be interesting to further investigate the significance of adipocytes apoA-I recycling in obesity.

Ectopic expression of $\beta$-ATPase on the plasma membrane of adipocytes was demonstrated to be involved in mediating the endocytosis and re-secretion of apoA-I [15]. Interestingly, the ectopic expression of $\beta$-ATPase 
was found to be increased during adipogenesis [68]. However, the physiological significance of $\beta$-ATPase in adipogenesis is not known. Our preliminary study observed that apoA-I could regulate adipocyte differentiation with a mechanism which is not clear. Therefore, further studies should be carried out to investigate apoA-I recycling at different stage of adipogenesis. Whether apoA-I recycling is involved in adipogenesis is an interesting question for further exploration.

\section{Transport of cellular miRNA}

It has been reported recently that HDL can transport microRNAs [69], which are short non-coding regulatory RNAs that modulate biological homeostasis by controlling gene expression through mRNA target and translational repression. Circulating miRNAs can be transported from donor cells to recipient cells and are viewed as a new class of biomarkers for a diverse set of diseases [70]. As the main protein component of HDL, apoA-I has been used for the systemic and specific delivery of small interfering RNA (siRNA) to hepatocytes in animal models [71]. It is interesting to hypothesize that apoA-I might acquire microRNAs during intracellular trafficking in adipocytes and deliver regulatory information to recipient cells when it is re-secreted into the plasma.

\section{Summary}

To sum up, uptake and resecretion of apoA-I by adipocytes were detected by using different apoA-I-labeling methods. Current studies on the mechanisms of apoA-I uptake by adipocytes support that it is a receptor mediated process which is ABCA1 independent but involves $\beta$-ATPase ectopically expressed on the plasma membrane. Besides, cAMP/PKA signaling regulates recycling of apoA-I by adipoctes, with the underlying mechanisms remain unclear. Whether apoA-I internalization is a clathrin and caveolae dependent process needs to be further studied. Although apoA-I recycling by adipocytes does not seem to play a major role in regulating adipose cholesterol metabolism under physiological conditions, it is likely that apoA-I recycling becomes important in pathological conditions when cholesterol mobilization is stimulated. Because cholesterol and TG metabolism are coupled in adipose tissue and recent recognition of the relationship between apoA-I and obesity, the physiological relevance of apoA-I recycling to obesity and adipogenesis are interesting questions to be answered. During intracellular transport, apoA-I may function as a vehicle through interaction with other proteins. The cargo is not restricted to intracellular cholesterol but may also include miRNAs, which modulate gene expression of target cells when apoA-I-miRNAs complex are re-secreted into the circulation by adipocytes.

\section{Abbreviations}

ABCA1: ATP-binding cassette transporter A1; AMPK: adenosine

5'-monophosphate-activated protein kinase; apoA-l: apoliprotein A-l; Asp: aspartate; CLPP: cytosolic lipid protein particles; Cys: cysteine; ER: endoplasmic reticulum; MCP-1: monocyte chemotactic protein-1; MDC: monodansyl cadaverine; MEFs: mouse embryonic fibroblast; NADPH oxidase: nicotinamide adenine dinucleotide phosphate-oxidase; OSBP: oxysterol binding protein; PI3K: phosphatidylinositol 3-kinase; PKA: protein kinase A; pka-apoA-l: recombinant apoA-I containing a phosphorylation site; PM: plasma membrane; Pro: proline; ROS: reactive oxygen species; SSA3: serum amyloid A3; StAR: steroidogenic acute regulatory; StARD3: START domain protein 3; START: StAR-related lipid transfer; $\beta$-ATPase: $\beta$ subunit of ATP synthase.

\section{Competing interests}

The authors declare that they have no competing interests.

\section{Authors' contribution}

DP conceived of the scope of the review and helped draft the manuscript. $S W$ and $Y-Y$ were involved in the accumulation of the relevant references and drafted the manuscript. All authors read and approved the final manuscript.

\section{Funding}

This study was supported by a grant from National Natural Science Foundation of China (Project No. 81370393).

\section{Author details}

'Department of Cardiology, The Second Xiangya Hospital of Central South University, Changsha, Hunan, China. ${ }^{2}$ The Second Xiangya Hospital of Central South University, Changsha, Hunan, China.

Received: 19 November 2015 Accepted: 11 February 2016

Published online: 24 February 2016

\section{References}

1. Higuchi K, Law SW, Hoeg JM, Schumacher UK, Meglin N, Brewer Jr HB. Tissue-specific expression of apolipoprotein A-I (ApoA-l) is regulated by the 5'-flanking region of the human ApoA-I gene. J Biol Chem. 1988;263:18530-6.

2. Zhang Y, McGillicuddy FC, Hinkle CC, O'Neill S, Glick JM, Rothblat GH, et al. Adipocyte modulation of high-density lipoprotein cholesterol. Circulation. 2010;121:1347-55

3. Chung S, Sawyer JK, Gebre AK, Maeda N, Parks JS. Adipose tissue ATP binding cassette transporter A1 contributes to high-density lipoprotein biogenesis in vivo. Circulation. 2011;124:1663-72.

4. Z Zhang Q, Zhang Y, Feng H, Guo R, Jin L, Wan R, et al. High density lipoprotein (HDL) promotes glucose uptake in adipocytes and glycogen synthesis in muscle cells. PLoS One. 2011;6:e23556.

5. Peterson SJ, Drummond G, Kim DH, Li M, Kruger AL, Ikehara S, et al. L-4 F treatment reduces adiposity, increases adiponectin levels, and improves insulin sensitivity in obese mice. J Lipid Res. 2008;49:1658-69.

6. Van Linthout S, Foryst-Ludwig A, Spillmann F, Peng J, Feng Y, Meloni M, et al. Impact of HDL on adipose tissue metabolism and adiponectin expression. Atherosclerosis. 2010;210:438-44.

7. Umemoto T, Han CY, Mitra P, Averill MM, Tang C, Goodspeed L, et al. Apolipoprotein $\mathrm{Al}$ and high-density lipoprotein have anti-inflammatory effects on adipocytes via cholesterol transporters: ATP-binding cassette A-1, ATP-binding cassette G-1, and scavenger receptor B-1. Circ Res. 2013;112: 1345-54.

8. Vanella L, Li M, Kim D, Malfa G, Bellner L, Kawakami T, et al. ApoA1: mimetic peptide reverses adipocyte dysfunction in vivo and in vitro via an increase in heme oxygenase (HO-1) and Wnt10b. Cell Cycle. 2012;11:706-14.

9. Mineo C, Shaul PW. Regulation of signal transduction by HDL. J Lipid Res. 2013:54:2315-24.

10. Nofer JR. Signal transduction by HDL: agonists, receptors, and signaling cascades. Handb Exp Pharmacol. 2015;224:229-56.

11. Lorenzi I, von Eckardstein A, Cavelier C, Radosavljevic S, Rohrer L. Apolipoprotein A-I but not high-density lipoproteins are internalised by RAW macrophages: roles of ATP-binding cassette transporter A1 and scavenger receptor BI. J Mol Med (Berl). 2008;86:171-83. 
12. Faulkner LE, Panagotopulos SE, Johnson JD, Woollett LA, Hui DY, Witting SR, et al. An analysis of the role of a retroendocytosis pathway in ABCA1-mediated cholesterol efflux from macrophages. J Lipid Res. 2008;49:1322-32.

13. Azuma Y, Takada M, Shin HW, Kioka N, Nakayama K, Ueda K. Retroendocytosis pathway of ABCA1/apoA-I contributes to HDL formation. Genes Cells. 2009;14:191-204

14. Verghese PB, Arrese EL, Howard AD, Soulages JL. Brefeldin A inhibits cholesterol efflux without affecting the rate of cellular uptake and re-secretion of apolipoprotein A-1 in adipocytes. Arch Biochem Biophys. 2008;478:161-6.

15. Howard AD, Verghese PB, Arrese EL, Soulages JL. The beta-subunit of ATP synthase is involved in cellular uptake and resecretion of apoA-I but does not control apoA-l-induced lipid efflux in adipocytes. Mol Cell Biochem. 2011;348:155-64.

16. Ruan X, Li Z, Zhang Y, Yang L, Pan Y, Wang Z, et al. Apolipoprotein A-I possesses an anti-obesity effect associated with increase of energy expenditure and up-regulation of UCP1 in brown fat. J Cell Mol Med. 2011;15:763-72

17. Martinez LO, Jacquet S, Esteve JP, Rolland C, Cabezon E, Champagne E, et al. Ectopic beta-chain of ATP synthase is an apolipoprotein A-I receptor in hepatic HDL endocytosis. Nature. 2003;421:75-9.

18. Radojkovic C, Genoux A, Pons V, Combes G, de Jonge H, Champagne E, et al. Stimulation of cell surface F1-ATPase activity by apolipoprotein A-I inhibits endothelial cell apoptosis and promotes proliferation. Arterioscler Thromb Vasc Biol. 2009;29:1125-30.

19. Malaval C, Laffargue M, Barbaras R, Rolland C, Peres C, Champagne E, et al. RhoA/ROCK I signalling downstream of the P2Y13 ADP-receptor controls HDL endocytosis in human hepatocytes. Cell Signal. 2009;21:120-7.

20. Gordon Jl, Sims HF, Lentz SR, Edelstein C, Scanu AM, Strauss AW. Proteolytic processing of human preproapolipoprotein A-I. A proposed defect in the conversion of pro A-I to A-I in Tangier's disease. J Biol Chem. 1983;258: 4037-44.

21. Saku K, von Eckardstein A, Zhang B, Liu R, Jimi S, Ou J, et al. In vivo kinetics of human apolipoprotein A-I variants in rabbits. Eur J Clin Invest. 1999;29:196-203.

22. Isacchi A, Sarmientos P, Lorenzetti R, Soria M. Mature apolipoprotein Al and its precursor proApoAl: influence of the sequence at the 5 ' end of the gene on the efficiency of expression in Escherichia coli. Gene. 1989;81:129-37.

23. McGuire KA, Davidson WS, Jonas A. High yield overexpression and characterization of human recombinant proapolipoprotein A-I. J Lipid Res. 1996;37:1519-28.

24. Schmidt HH, Remaley AT, Stonik JA, Ronan R, Wellmann A, Thomas F, et al. Carboxyl-terminal domain truncation alters apolipoprotein A-I in vivo catabolism. J Biol Chem. 1995;270:5469-75.

25. Pyle LE, Sawyer WH, Fujiwara Y, Mitchell A, Fidge NH. Structural and functional properties of full-length and truncated human proapolipoprotein Al expressed in escherichia coli. Biochemistry. 1996;35:12046-52.

26. Safi W, Maiorano JN, Davidson WS. A proteolytic method for distinguishing between lipid-free and lipid-bound apolipoprotein A-I. J Lipid Res. 2001;42:864-72.

27. Panagotopulos SE, Witting SR, Horace EM, Nicholas Maiorano J, Sean Davidson W. Bacterial expression and characterization of mature apolipoprotein A-I. Protein Expr Purif. 2002;25:353-61.

28. Ryan RO, Forte TM, Oda MN. Optimized bacterial expression of human apolipoprotein A-I. Protein Expr Purif. 2003;27:98-103.

29. Braschi S, Neville TA, Maugeais C, Ramsamy TA, Seymour R, Sparks DL. Role of the kidney in regulating the metabolism of HDL in rabbits: evidence that iodination alters the catabolism of apolipoprotein A-I by the kidney. Biochemistry. 2000;39:5441-9.

30. Anderson RG, Brown MS, Goldstein JL. Role of the coated endocytic vesicle in the uptake of receptor-bound low density lipoprotein in human fibroblasts. Cell. 1977;10:351-64.

31. Takahashi Y, Smith JD. Cholesterol efflux to apolipoprotein Al involves endocytosis and resecretion in a calcium-dependent pathway. Proc Natl Acad Sci U S A. 1999;96:11358-63.

32. Parton RG, del Pozo MA. Caveolae as plasma membrane sensors, protectors and organizers. Nat Rev Mol Cell Biol. 2013;14:98-112.

33. Wang F, Gu HM, Zhang DW. Caveolin-1 and ATP binding cassette transporter A1 and G1-mediated cholesterol efflux. Cardiovasc Hematol Disord Drug Targets. 2014;14:142-8

34. Le Lay S, Rodriguez M, Jessup W, Rentero C, Li Q, Cartland S, et al. Caveolin1-mediated apolipoprotein A-I membrane binding sites are not required for cholesterol efflux. PLoS One. 2011;6:e23353.
35. Wang N, Silver DL, Thiele C, Tall AR. ATP-binding cassette transporter A1 (ABCA1) functions as a cholesterol efflux regulatory protein. J Biol Chem. 2001;276:23742-7.

36. Neufeld EB, Stonik JA, Demosky Jr SJ, Knapper CL, Combs CA, Cooney A et al. The ABCA1 transporter modulates late endocytic trafficking: insights from the correction of the genetic defect in Tangier disease. J Biol Chem. 2004;279:15571-8.

37. Smith JD, Waelde C, Horwitz A, Zheng P. Evaluation of the role of phosphatidylserine translocase activity in ABCA1-mediated lipid efflux. J Biol Chem. 2002;277:17797-803.

38. Chen W, Wang N, Tall AR. A PEST deletion mutant of ABCA1 shows impaired internalization and defective cholesterol efflux from late endosomes. J Biol Chem. 2005:280:29277-81.

39. Norata GD, Callegari E, Marchesi M, Chiesa G, Eriksson P, Catapano AL. Highdensity lipoproteins induce transforming growth factor-beta2 expression in endothelial cells. Circulation. 2005;111:2805-11.

40. Fabre AC, Vantourout $P$, Champagne E, Terce F, Rolland C, Perret B, et al. Cell surface adenylate kinase activity regulates the F(1)-ATPase/P2Y (13)mediated HDL endocytosis pathway on human hepatocytes. Cell Mol Life Sci. 2006;63:2829-37.

41. Zhang LH, Kamanna VS, Zhang MC, Kashyap ML. Niacin inhibits surface expression of ATP synthase beta chain in HepG2 cells: implications for raising HDL. J Lipid Res. 2008;49:1195-201.

42. Haidar B, Denis M, Marcil M, Krimbou L, Genest Jr J. Apolipoprotein A-I activates cellular CAMP signaling through the ABCA1 transporter. J Biol Chem. 2004:279:9963-9.

43. Hu YW, Ma X, Li XX, Liu XH, Xiao J, Mo ZC, et al. Eicosapentaenoic acid reduces $A B C A 1$ serine phosphorylation and impairs $A B C A 1$-dependent cholesterol efflux through cyclic AMP/protein kinase A signaling pathway in THP-1 macrophage-derived foam cells. Atherosclerosis. 2009;204:e35-43.

44. Haidar B, Denis M, Krimbou L, Marcil M, Genest Jr J. CAMP induces ABCA1 phosphorylation activity and promotes cholesterol efflux from fibroblasts. J Lipid Res. 2002;43:2087-94.

45. See RH, Caday-Malcolm RA, Singaraja RR, Zhou S, Silverston A, Huber MT, et al. Protein kinase A site-specific phosphorylation regulates ATP-binding cassette A1 (ABCA1)-mediated phospholipid efflux. J Biol Chem. 2002:277: 41835-42.

46. Smith JD, Miyata M, Ginsberg M, Grigaux C, Shmookler E, Plump AS. Cyclic AMP induces apolipoprotein E binding activity and promotes cholesterol efflux from a macrophage cell line to apolipoprotein acceptors. J Biol Chem. 1996:271:30647-55.

47. Denis M, Landry YD, Zha X. ATP-binding cassette A1-mediated lipidation of apolipoprotein A-I occurs at the plasma membrane and not in the endocytic compartments. J Biol Chem. 2008;283:16178-86.

48. Vedhachalam C, Duong PT, Nickel M, Nguyen D, Dhanasekaran P, Saito H, et al. Mechanism of ATP-binding cassette transporter A1-mediated cellular lipid efflux to apolipoprotein A-I and formation of high density lipoprotein particles. J Biol Chem. 2007;282:25123-30

49. Wang S, Gulshan K, Brubaker G, Hazen SL, Smith JD. ABCA1 mediates unfolding of apolipoprotein AI N terminus on the cell surface before lipidation and release of nascent high-density lipoprotein. Arterioscler Thromb Vasc Biol. 2013;33:1197-205.

50. Bencharif K, Hoareau L, Murumalla RK, Tarnus E, Tallet F, Clerc RG, et al. Effect of apoA-I on cholesterol release and apoE secretion in human mature adipocytes. Lipids Health Dis. 2010;9:75.

51. Verghese PB, Arrese EL, Soulages JL. Stimulation of lipolysis enhances the rate of cholesterol efflux to HDL in adipocytes. Mol Cell Biochem. 2007:302:241-8.

52. Le Lay S, Robichon C, Le Liepvre X, Dagher G, Ferre P, Dugail I. Regulation of $A B C A 1$ expression and cholesterol efflux during adipose differentiation of 3 T3-L1 cells. J Lipid Res. 2003:44:1499-507.

53. Krause BR, Hartman AD. Adipose tissue and cholesterol metabolism. J Lipid Res. 1984:25:97-110.

54. Schreibman PH, Dell RB. Human adipocyte cholesterol. Concentration, localization, synthesis, and turnover. J Clin Invest. 1975;55:986-93.

55. DeOgburn R, Leite JO, Ratliff J, Volek JS, McGrane MM, Fernandez ML. Effects of increased dietary cholesterol with carbohydrate restriction on hepatic lipid metabolism in Guinea pigs. Comp Med. 2012;62:109-15.

56. Prattes S, Horl G, Hammer A, Blaschitz A, Graier WF, Sattler W, et al. Intracellular distribution and mobilization of unesterified cholesterol in adipocytes: triglyceride droplets are surrounded by cholesterol-rich ER-like surface layer structures. J Cell Sci. 2000;113(Pt 17):2977-89. 
57. Maxfield FR, Wustner D. Intracellular cholesterol transport. J Clin Invest. 2002;110:891-8.

58. Soccio RE, Breslow JL. Intracellular cholesterol transport. Arterioscler Thromb Vasc Biol. 2004:24:1150-60.

59. Ito J, Michikawa M. ApoA-//HDL Generation and Intracellular Cholesterol Transport through Cytosolic Lipid-Protein Particles in Astrocytes. J Lipids. 2014;2014:530720.

60. Rigotti A, Cohen DE, Zanlungo S. STARTing to understand MLN64 function in cholesterol transport. J Lipid Res. 2010;51:2015-7.

61. Soccio RE, Breslow JL. StAR-related lipid transfer (START) proteins: mediators of intracellular lipid metabolism. J Biol Chem. 2003;278:22183-6.

62. Mesmin B, Antonny B, Drin G. Insights into the mechanisms of sterol transport between organelles. Cell Mol Life Sci. 2013;70:3405-21.

63. Mesmin B, Bigay J, Moser von Filseck J, Lacas-Gervais S, Drin G, Antonny B. A four-step cycle driven by PI(4)P hydrolysis directs sterol/PI(4)P exchange by the ER-Golgi tether OSBP. Cell. 2013;155:830-43.

64. Peterson SJ, Kim DH, Li M, Positano V, Vanella L, Rodella LF, et al. The L-4 F mimetic peptide prevents insulin resistance through increased levels of HO-1, pAMPK, and pAKT in obese mice. J Lipid Res. 2009;50:1293-304.

65. Wei H, Averill MM, McMillen TS, Dastvan F, Mitra P, Subramanian S, et al. Modulation of adipose tissue lipolysis and body weight by high-density lipoproteins in mice. Nutr Diabetes. 2014;4:e108.

66. Lindahl M, Petrlova J, Dalla-Riva J, Wasserstrom S, Rippe C, Domingo Espin J, et al. ApoA-I Milano stimulates lipolysis in adipose cells independently of CAMP/PKA activation. J Lipid Res. 2015;56(12):2248-59.

67. Ibanez B, Giannarelli C, Cimmino G, Santos-Gallego CG, Alique M, Pinero A, et al. Recombinant HDL(Milano) exerts greater anti-inflammatory and plaque stabilizing properties than HDL(wild-type). Atherosclerosis. 2012;220:72-7.

68. Arakaki N, Kita T, Shibata H, Higuti T. Cell-surface H+-ATP synthase as a potential molecular target for anti-obesity drugs. FEBS Lett. 2007;581:3405-9.

69. Vickers KC, Palmisano BT, Shoucri BM, Shamburek RD, Remaley AT MicroRNAs are transported in plasma and delivered to recipient cells by high-density lipoproteins. Nat Cell Biol. 2011;13:423-33.

70. Simons M, Raposo G. Exosomes-vesicular carriers for intercellular communication. Curr Opin Cell Biol. 2009;21:575-81.

71. Kim SI, Shin D, Choi TH, Lee JC, Cheon GJ, Kim KY, et al. Systemic and specific delivery of small interfering RNAs to the liver mediated by apolipoprotein A-I. Mol Ther. 2007;15:1145-52.

\section{Submit your next manuscript to BioMed Central and we will help you at every step:}

- We accept pre-submission inquiries

- Our selector tool helps you to find the most relevant journal

- We provide round the clock customer support

- Convenient online submission

- Thorough peer review

- Inclusion in PubMed and all major indexing services

- Maximum visibility for your research

Submit your manuscript at www.biomedcentral.com/submit

) Biomed Central 